\title{
CONDUCTANCE MODULATION IN SEMICONDUCTOR MICROSTRUCTURE WITH PERIODIC ARRAY OF MICROMAGNETS
}

\author{
I. Tralle \\ Institute of Physics, University of Rzeszów \\ Al. Rejtana 16A, 35-310 Rzeszów, Poland
}

\begin{abstract}
A simple theory for the Aharonov-Bohm-like effect (dynamical phase) in semiconductor microstructure, is presented in a paper, assuming a "ballistic spin transport". If in one of the arms of microstructure there is an additional periodic magnetic field, the spin wave function acquires a phase shift due to additional spin precession about that field. By introducing quaternion representation of the elements of propagation matrices, one can calculate the transition coefficient of the structure. It is shown that the deep modulation of the conductance could be achieved.
\end{abstract}

PACS numbers: $72.15 . \mathrm{Gd}, 73.50 . \mathrm{Bk}$

\section{Introduction}

A lot of interesting physical effects are due to quantum interference in the presence of topologically nontrivial electromagnetic fields. Among them are the Aharonov-Bohm effect [1], the Dirac monopole [2], and the quantization of flux in superconductivity [3]. Oscillatory magnetoresistance due to the Aharonov-Bohm effect has been observed in small metallic rings [4] and MBE grown double quantum wells [5]. The origin of these oscillations ( $h / e$ as well as $h / 2 e)$ is now well understood due to the efforts of a number of researchers. These works were concerned however to the diffusive transport of charge carriers. In their well-known paper [6], Datta and Bandyopadhyay considered a ballistic transport and have shown the possibility (in principle) to approach even $100 \%$ conductance modulation in a magnetic field. The conditions to which the microstructure has to obey are however very strict. In particular, there should be a "single mode" regime which by all means is difficult to be achieved. It should be noted that so far the researchers considered only the Hamiltonian $H=(\boldsymbol{p}+(e / c) \boldsymbol{A})^{2} / 2 m^{*}+U(y)$, where $U(y)$ is the energy corresponding to transverse motion, and almost nobody takes into account the spin-part $\mu_{\mathrm{B}} \sigma \boldsymbol{B}$ of the Hamiltonian ( $\mu_{\mathrm{B}}$ is the Bohr magneton, $\sigma$ is the electron spin operator, $\boldsymbol{B}$ - magnetic field). The main reason for neglecting 
this term is that the Bohr magneton is very small and as a result, the energy of electron charge interaction with the external field is much greater than that of spin interaction. However, generally speaking, the spin-part of the electron wave function also can acquire a phase shift in course of electron evolution in magnetic field and hence cause the conduction to oscillate. The aim of the paper is to present a simple theory for such Aharonov-Bohm-like (dynamical phase) effect in semiconductor microstructure with periodic array of micromagnets.

\section{The model and necessary preliminaries}

Let us consider a generic microstructure with two end regions $(x<0$ and $x>L$ ) and a middle region $0 \leq x \leq L$ consisting of two channels (Fig. 1) similar to that one considered in [6]. The main difference however is that the external magnetic field $\boldsymbol{B}_{0}$ is in the plane of microstructure and in addition, on the upper surface of one of the channels there is a regular array of micromagnets similar to that of recently has been reported by von Klitzing et al. [7]. These micromagnets create within this channel an additional periodic magnetic field $\boldsymbol{B}_{1}$ of the form

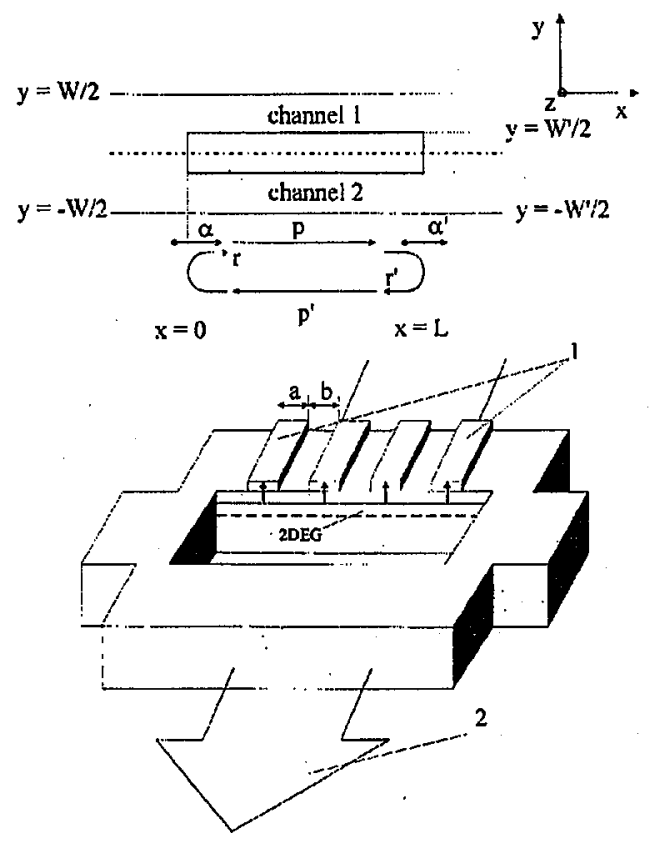

Fig. 1. Sketch of a two-channel semiconductor microstructure with the magnetic grating on the top of one of the channels. By means of $\alpha, \alpha^{\prime}, r, r^{\prime}$ the transmission and reflection matrices at the two junctions $x<0, x>L$ are indicated; $P, P^{\prime}$ stand for the propagation matrices in the middle region $(0<x<L) .1-$ micromagnets (one-dimensional ferromagnetic grating), 2 - external magnetic field $\boldsymbol{B}_{0}$. 


$$
\begin{aligned}
& \boldsymbol{B}_{1}\left(0,0, B_{1}\right)=B_{1}(x) \\
& \quad= \begin{cases}B_{1}[x-(n-1)] a, & (n+1) a \leq x \leq(n-1) a+b \\
0 & (n-1) a+b \leq x<n a, \quad n=1,2,3 \ldots\end{cases}
\end{aligned}
$$

Here $a$ is the width of the magnetic strip and $b$ is the spacing, so that $a+b$ is the period of magnetic grating. This means that the magnetic field $\boldsymbol{B}$ which affects the electron in the first arm of the structure is equal to $\boldsymbol{B}=\boldsymbol{B}_{0}$ while in the other one it is equal to $\boldsymbol{B}=\boldsymbol{B}_{0}+\boldsymbol{B}_{1}$.

Suppose the Hamiltonian of the electron is

$$
\begin{aligned}
& H=H_{0}+H_{1}, \\
& H_{0}=\left(1 / 2 m^{*}\right)[p-(e / c) \boldsymbol{A}]^{2}+U(\boldsymbol{r}), \quad H_{1}=-\mu_{\mathrm{B}} \boldsymbol{\sigma} \boldsymbol{B},
\end{aligned}
$$

where $m^{*}$ is the electron effective mass, $\boldsymbol{A}$ is vector potential corresponding to the magnetic field $\boldsymbol{B}, \mu_{\mathrm{B}}$ and $\sigma$ are Bohr magneton and the spin operator, respectively. We also take it for granted that $U(\boldsymbol{r})$ describes conduction bands bending due to the space charge and discontinuities of any band. Since $H_{0}$ does not depend on spin, the wave function is the direct product: $\Psi(r, s)=\varphi(r) \otimes \chi(s)$. Ever since for convenience we shall refer to $\varphi(r)$ as the "charge-part" of the total wave function keeping in mind that it corresponds to $H_{0}$ describing the charge-field interaction, while we shall refer to $\chi(s)$ as spin-part of the wave function related to $H_{1}$, spin part of the Hamiltonian $H$ in Eq. (2).

Let us introduce now the phase-relaxation length $L_{\varphi}^{(s)}$ for the spin part of the wave function just like it is usually introduced for the "charge-part" one, $L_{\varphi}^{(e)}$. Our main hypothesis is that the phase relaxation length $L_{\varphi}^{(s)}$ is much greater than $L_{\varphi}^{(e)}$. The reason is quite simple: the electron spin-phonon interaction is much smaller than electron charge-phonon interaction. However, there is a need to make some additional remarks here. As is known, as a rule rigid scatterers such as impurities and other defects of crystalline structure do not contribute to phase relaxation; only dynamical scatterers like lattice vibrations (phonons) do. But impurity scattering also can be phase randomizing if the impurity has an internal degree of freedom so that it can change its state. For example if magnetic impurities have an internal spin that fluctuates with time, the collisions with such impurities cause phase relaxation. Therefore, we suppose that there are no such impurities here.

Now if we suppose the microstructure length $L$ is chosen to be $L_{\varphi}^{(s)}>L>$ $L_{\varphi}^{(e)}$, it would be possible to "wash out" the quantum interference related to phase coherence of "charge part" of the wave function retaining at the same time the phase coherence of the spin part one and hence, to reveal the corresponding conductance oscillations.

Let us now estimate the phase-relaxation length $L_{\varphi}^{(s)}$ which is defined by means of phase-relaxation time $\tau_{\varphi}^{(s)}, L_{\varphi}^{(s)}=\nu_{\mathrm{F}} \tau_{\varphi}^{(s)}$, where $\nu_{\mathrm{F}}$ is the Fermi velocity. Consider two-state quantum system (which we also shall refer to as subsystem $S$ ) with excitation energy $\varepsilon$ interacting with the phonon bath and identify the two states with "spin-up" and "spin-down" states of a spin in an external magnetic 
field. For simplicity we suppose the subsystem $S$ interaction with the phonons to be resonant, it means that only those modes of the phonon bath whose energy is equal to $\varepsilon$ interact with the two-level subsystem. Other degrees of freedom are taken into account by means of choosing other mean values of bath parameters to be equal to corresponding statistical average at given temperature. As a result, for a model of phonon bath we can take a great number $(N \gg 1)$ of identical non-interacting subsystems $b_{n}$ with excitation energy $\varepsilon$. Thus, the Hamiltonian of the entire system (subsystem $S+$ phonon bath) is

$$
H=\varepsilon\left(s^{\dagger} s+\sum_{n=1}^{N} b_{n}^{\dagger} b_{n}\right)
$$

where $s^{\dagger}, s$ are the Fermi creation and annihilation operators related to the excitations of subsystem $S$, while $b_{n}^{\dagger}, b_{n}$ are Bose creation and annihilation operators related to the excitations of the $n$-th subsystem of phonon bath.

The two-level subsystem interaction with phonons can be described by the $H_{\text {int }}(t)$ as follows:

$$
H_{\text {int }}(t)=\sum_{n=1}^{N}\{\Theta[t-\tau(n-1)]-\Theta(t-\tau n)\} H_{n},
$$

where $\Theta$ is the step-like (unit-valued Heaviside) function, $H_{n}=\varepsilon_{\text {int }}\left(s^{\dagger} b_{n}+b_{n}^{\dagger} s\right)$ and $\varepsilon_{\text {int }}$ is the interaction energy. The physical meaning of (3) is that the subsystem $S$ interacts each time during interval $\tau$ with those subsystem $b_{n}$ which did not interact with $S$ during previous time interval.

Introduce now two probabilities $w_{1}(t)$ and $w_{2}(t)$ of the subsystem $S$ to be at a time $t$ in excited state and unexcited one, respectively. It is well known [8] that the steady state for such two-level subsystem corresponds to $w_{1}=w_{2}=1 / 2$. As it can be shown for the model described above, time $t$ which is needed for the subsystem $a$ to achieve the state of $w_{1}=w_{2}=1 / 2$ is equal to

$$
t=\left(\hbar^{2} / \tau \varepsilon_{\text {int }}^{2}\right) \ln 2 \tanh (\beta \varepsilon / 2), \quad \beta=1 / k_{\mathrm{B}} T,
$$

where $k_{\mathrm{B}}$ - the Boltzmann constant, $T$ is the temperature.

Since the two levels of the subsystem $S$ correspond to the "spin-up" and "spin-down" states, the steady state corresponds to the redistribution of initially non-equilibrium spin distribution due to spin flips and hence, to the total destruction of spin coherence. Thus, time $t$ is the spin-relaxation time which can be attributed to phase-relaxation time $\tau_{\varphi}^{(s)}$, because it relates to the phase coherence destruction caused by inelastic scattering.

In order to estimate $t \sim \tau_{\varphi}^{(s)}$ by means of Eq. (4), we should estimate at first the interaction energy $\varepsilon_{\text {int }}$. Thus, considering the spin flips under the scattering by acoustic phonons, one should take into account the spin-orbit interaction [9]. If we take it to be $\varepsilon_{\operatorname{int}} \sim(e \hbar / 2 m c)^{2}\left(a^{*}\right)^{3}$, where $c$ is light velocity, $a^{*}-$ the Bohr radius, $\tau$ is of about $10^{-15} \mathrm{~s}$, magnetic field is of the $1 \mathrm{~T}$ while the temperature of about $5 \mathrm{~K}$, for the time $t=\tau_{\varphi}^{(s)}$ by (4) we have $\approx 2.2 \times 10^{-10} \mathrm{~s}$. The values of spin relaxation time measured for the $\mathrm{A}_{3} \mathrm{~B}_{5}$ semiconductors are widely ranged 
from $10^{-12}$ to $10^{-7} \mathrm{~s}[10]$ and hence we can conclude that our estimation for $\tau_{\varphi}^{(s)}$ is quite reasonable. Anyway, we can take it for certain that $\tau_{\varphi}^{(s)} \gg \tau_{\varphi}^{(e)}$. Indeed, the experiments show [11] that at $5 \mathrm{~K}$ the phase relaxation time $\tau_{\varphi}^{(e)}$ is of about $1.6 \times 10^{-12} \mathrm{~s}$ and as a result, $L_{\varphi}^{(s)} \gg L_{\varphi}^{(e)}$ and hence, the length of the structure $L$ can be chosen to be $L_{\varphi}^{(s)}>L>L_{\varphi}^{(e)}$.

\section{Calculation of the transmission coefficient}

The current $I$, through the structure considered in the previous section, for a small applied potential $V$, can be written as $[6,12]$ :

$$
I=\frac{2 e}{h} \int \mathrm{d} E \int\left(w_{z} \mathrm{~d} k_{z} / 2 \pi\right)[f(E)-f(E+e V)] \sum_{n^{\prime}, n^{\prime \prime}}\left|T_{n^{\prime}, n^{\prime \prime}}\right|^{2} .
$$

Here $w_{z}$ is the width of the structure in the $z$-direction, $T_{n^{\prime}, n^{\prime \prime}}$ is the transmission coefficient from the state $n^{\prime}$ in the left end to the state $n^{\prime \prime}$ in the right end, $E$ and $k_{z}$ are the energy and the transverse wave vector of the electrons as they enter from the left end. Let us suppose for a moment that the structure length is smaller than the phase relaxation length; then transport is said to be coherent and one calculates the transmission coefficient starting from the Schrödinger equation with the Hamiltonian (2). It is well known [12] that a coherent conductor can be characterised at each energy by an $S$-matrix that relates the outgoing wave amplitudes to the incoming wave amplitudes at the different leads. Thus, the transmission coefficient $T_{n^{\prime}, n^{\prime \prime}}$ can be obtained by taking the squared magnitude of the corresponding element of the $S$-matrix. Taking into account the relation $\Psi(r, s)=\varphi(r) \otimes \chi(s)$ and using the next property of the direct product $(A \otimes B)(C \otimes D)=A C \otimes B D$, one can demonstrate that $T_{n^{\prime}, n^{\prime \prime}}=T_{k^{\prime}, k^{\prime \prime}} \otimes T_{\sigma^{\prime}, \sigma^{\prime \prime}}$ where subscripts $k^{\prime}, k^{\prime \prime}$ are related to the states of $H_{0}$, while $\sigma^{\prime}, \sigma^{\prime \prime}$ - to the states of $H_{1}$.

Now take into account that the length of the structure $L$ is greater than $L_{\varphi}^{(e)}$. Then, dividing the structure into sections of the length smaller than $L_{\varphi}^{(e)}$, one can combine the successive scatters [12] and treat the transport through the states $k^{\prime}, k^{\prime \prime}$ as incoherent while the transport through the states $\sigma^{\prime}, \sigma^{\prime \prime}$ as the coherent one, because $L<L_{\varphi}^{(s)}$. As a result we have $T_{n^{\prime}, n^{\prime \prime}}=\langle T\rangle T_{\sigma^{\prime}, \sigma^{\prime \prime}}$, where $\langle T\rangle$ is the transition coefficient which does not depend on the phase relations between the states of $H_{0}$ in the left end and in the right one of the structure. Therefore, in accordance with the assumptions above, there are two states ("spin-up" and "spin-down") to consider in end regions, while in the middle region there are four states corresponding to the channels 1 and 2. Let us concentrate on the calculation of $T_{\sigma^{\prime}, \sigma^{\prime \prime}}$. Dropping the subscripts $\sigma^{\prime}, \sigma^{\prime \prime}$, one can write down the next expression for the transmission coefficient $T$ [13]:

$$
T=\alpha^{\prime}\left[I-P r P^{\prime} r^{\prime}\right]^{-1} P \alpha
$$

Here $I$ is the unit matrix, $\alpha$ is $4 \times 1$ matrix describing the transmission from the left end into the two channels while $\alpha^{\prime}$ is a $1 \times 4$ matrix describing the transmission from the channels into the right end. Similarly, $r$ and $r^{\prime}$ are $4 \times 4$ matrices describing the reflections at the two junctions from the channels back into 
the channels. In accordance with the consideration given in Sec. 2, there are no spin-flip transitions and hence, only the next matrix elements of $r$ are nonzero: $r_{11}, r_{13}, r_{22}, r_{24}, r_{31}, r_{33}, r_{42}, r_{44}$ (the same concerning matrix elements of the matrix $r^{\prime}$ ).

In order to construct $P$ and $P^{\prime}$, the matrices which describe forward and reverse propagation of electron wave through the channels 1 and 2 , it is necessary to note that the spin-parts of the wave functions acquire the phase factors due to spin precession about $\boldsymbol{B}$-axis. Since magnetic fields in the channels are different, these phase factors are also different. If one treats the states "spin-up" and "spin-down" as the two opposite points on a unit sphere $S^{2}$ which can be transformed one into another under rotation by an angle $\varphi= \pm \pi$ about some axis $e_{1}$ then the matrix elements describing the phase shifts in the two channels can be written as

$$
\begin{aligned}
& P_{ \pm 1}=\exp \left( \pm \mathrm{i} e_{1} \varphi\right) \exp \left(\mathrm{i} e_{2} \theta_{1}\right), \quad P_{ \pm 1}^{\prime}=\exp \left( \pm \mathrm{i} e_{1} \varphi\right) \exp \left(-\mathrm{i} e_{2} \theta_{1}\right) \\
& P_{ \pm 2}=\exp \left( \pm \mathrm{i} e_{1} \varphi\right) \exp \left(\mathrm{i} e_{2} \theta_{2}\right), \quad P_{ \pm 2}^{\prime}=\exp \left( \pm \mathrm{i} e_{1} \varphi\right) \exp \left(\mathrm{i} e_{2} \theta_{2}\right)
\end{aligned}
$$

Here we also formally introduced $\boldsymbol{e}_{2}$-axis which is a unit vector of the precession axis; $+e_{2}$ corresponds to the electron propagation from $x=0$ to $x=L$, while $-e_{2}$ corresponds to reverse propagation, $\theta_{1}$ and $\theta_{2}$ are the phases acquired by the spin-part of the wave functions in the channels 1 and 2, respectively.

The idea of (6) is to express the elements of the matrices $P$ and $P^{\prime}$ as the two rotations about two independent axis. Then, these objects are nothing but the unitary quaternions; these quaternions make four-dimensional real vector space and since two channels 1 and 2 are supposed to be isolated, the matrices $P$ and $P^{\prime}$ are diagonal $4 \times 4$ matrices with the diagonal elements defined by (6). After a great deal of algebra, we have:

$$
\begin{aligned}
& |T|^{2}=\left|a_{1}\right|^{2}+\left|a_{2}\right|^{2}+\left|a_{3}\right|^{2}+\left|a_{4}\right|^{2}+\left(a_{1}^{*} a_{3}+a_{1} a_{3}^{*}+a_{2}^{*} a_{4}+a_{2} a_{4}^{*}\right) \\
& +\left(a_{1}^{*} a_{2}+a_{1} a_{2}^{*}+a_{2}^{*} a_{3}+a_{2} a_{3}^{*}+a_{3}^{*} a_{4}+a_{3} a_{4}^{*}\right) \cos \Delta \theta, \quad \Delta \theta=\theta_{1}-\theta_{2},
\end{aligned}
$$

where $a_{i}(i=1,2,3,4)$ do not depend on $\theta_{1}, \theta_{2}$ and are the complicated functions of $r_{i j}, r_{i j}^{\prime}, \alpha_{i}, \alpha_{i}^{\prime}$. Therefore, the problem now is to calculate the additional phase shift $\Delta \theta=\theta_{1}-\theta_{2}$ which arises due to the precession of electron spin in the periodic magnetic field of micromagnets.

\section{Calculation of the phase shift}

Consider the non-relativistic motion of the particle (electron) with the spin $s=1 / 2$ in a two-component magnetic field: $\boldsymbol{B}=\boldsymbol{B}_{0}+\boldsymbol{B}_{1}, \boldsymbol{B}_{0}=\left(0, B_{0}, 0\right)$ and $\boldsymbol{B}_{1}=$ $\left(0,0, B_{1}(x)\right)$, where $B_{1}(x)$ is given by Eq. (1). Thus, we can treat the mean value of magnetic moment of the electron moving within the channels of microstructure as the classical quantity $\boldsymbol{P}=\langle\sigma\rangle$, its evolution under the magnetic field being defined by the equation

$$
\frac{\mathrm{d} \boldsymbol{P}}{\mathrm{d} t}=\gamma[\boldsymbol{P}, \boldsymbol{B}],
$$

where $\gamma=e / m c$ is the electron gyromagnetic constant.

In other words, vector $\boldsymbol{P}$ can be treated as classical magnetic top and if this classical top having the initial orientation $\boldsymbol{P}_{0}=\left(P_{x}^{0}, P_{y}^{0}, P_{z}^{0}\right)$ enters magnetic field 
$\boldsymbol{B}=\left(B_{x}, B_{y}, B_{z}\right)$, it begins to precess about magnetic field with the frequency $\Omega=\gamma B$, where $B=\left(B_{x}, B_{y}, B_{z}\right)^{1 / 2}$. In the reference frame of electron moving with the velocity $\nu$ in the space domain occupied by the magnetic fields $\boldsymbol{B}_{0}, \boldsymbol{B}_{1}$, the varying field of the frequency $\omega(\nu)=2 \pi \nu /(a+b)$, where $(a+b)$ is the period of magnetic grating, affects the electron. This oscillating field in the reference frame rotating with the frequency $\omega(\nu)$ is of the form

$$
B_{x}=0, \quad B_{y}=B_{0}-\omega(\nu) / \gamma, \quad B_{z}=B_{1}(x) .
$$

Let us introduce now the phase of the precessing spin by means of the formula

$$
\theta(\nu, x)=\left(\mu_{\mathrm{B}} / \hbar\right) \int_{0}^{x} B(\nu, t) \mathrm{d} t=\gamma \int_{0}^{x} B(\nu, t) \mathrm{d} t,
$$

and take into account that the fields $\boldsymbol{B}_{0}, \boldsymbol{B}_{1}$ are uniform. Therefore, the field $\boldsymbol{B}$ depends only on $\nu$ but not on $x$ and the phase of precessing spin depends on $t$ linearly: $\theta(\nu)=\gamma B(\nu) t$. Now the calculation of the phase shift $\Delta \theta$ can easily be done. Moreover, it is clear that under certain conditions including appropriate structure length $L=m(a+b), m=2, \ldots N$, electron velocity and the values of magnetic fields $\boldsymbol{B}_{0}, \boldsymbol{B}_{1}$, the phase shift $\Delta \theta=\theta_{2}-\theta_{1}$ can be multiple of $\pi / 2$. Indeed,

$$
\Delta \theta=\theta_{2}-\theta_{1}=(n+1 / 2) \pi=\gamma\left(\sqrt{B_{y}^{2}+B_{z}^{2}}-B_{0}\right) L / \nu, \quad n=0,1,2, \ldots
$$

where $B_{y}=B_{0}-\omega(\nu) / \gamma=B_{0}-\pi \nu / \gamma(a+b), B_{z}=B_{1}$.

If the values of $B_{z}, L, \nu$ are given, the value of $B_{0}$ can be easily calculated

$$
B_{0}=(1 / 2)\left[\left(1-\frac{(n+1 / 2)^{2}}{m^{2}}\right) \frac{\pi \nu}{\gamma(a+b)}+\frac{B_{1}^{2}}{\pi \nu} \gamma(a+b)\right],
$$

and we can see that $\Delta \theta=\theta_{2}-\theta_{1}=f\left(B_{0}, B_{1}, \nu\right)$, the function of $B_{0}, B_{1}, \nu$. That is, the phase shift, generally speaking, is different for the electrons with different velocities. At first sight it makes matters worse because it means that the "interference pattern" should be blurred. One should remember however the temperature is considered to be sufficiently low. That is the electron distribution function $f(E)=\Theta\left(E_{\mathrm{F}}-E\right)$ and $\nu=\nu_{\mathrm{F}}$, where $\Theta(\ldots)$ is the Heaviside step-like function, $E_{\mathrm{F}}, \nu_{\mathrm{F}}$ are the Fermi energy and Fermi velocity, respectively. Therefore, the calculation by means of Eq. (5) now can easily be done and we have

$$
I=(2 e / \hbar) K\left[A+D \cos \Delta \theta\left(\nu_{\mathrm{F}}\right)\right] \text {, }
$$

where $K, A, D$ are the coefficients dependent on the peculiarities of structure. Now it is clear that changing $B_{0}$, one can approach very deep modulation of the conductance and since $A \sim D$, the "contrast" of the "interference pattern" is defined only by the ratio $\sqrt{\left(E_{\mathrm{F}}-k_{\mathrm{B}} T\right) / E_{\mathrm{F}}}$ which at the temperature of about $40 \mathrm{~K}$ is of the order of $90 \%$.

\section{References}

[1] Y. Aharonov, D. Bohm, Phys. Rev. 115, 485 (1959).

[2] P.A.M. Dirac, Proc. R. Soc. Lond. A 133, 60 (1931).

[3] N. Byers, C.N. Yang, Phys. Rev. Lett. 7, 46 (1961).

[4] Mesoscopic Phenomena in Solids, Eds. B.L. Al'tshuler, P.A. Lee, R.A. Webb, North-Holland, New York 1991. 
[5] D. Mailly, C. Chapelier, A. Benoit, Phys. Rev. Lett. 70, 2020 (1993).

[6] S. Datta, S. Bandyopadhyay, Phys. Rev. Lett. 58, 717 (1987).

[7] P.D. Ye, D. Weiss, R.R. Gerhards, M. Seeger, K. von Klitzing, K. Ebert, H. Nickel, Phys. Rev. Lett. 74, 3013 (1995).

[8] A.S. Davydov, Quantum Mechanics, Nauka, Moskva 1973, p. 488 (in Russian).

[9] V.F. Gantmacher, Y.B. Levinson, Carrier Scattering in Metals and Semiconductors, North-Holland, New York 1987.

[10] R.R. Parsons, Can. J. Phys. 49, 1850 (1971).

[11] K.K. Choi, D.C. Tsui, K. Alavi, Phys. Rev. B 36, 7751 (1987).

[12] S. Datta, Electronic Transport in Mesoscopic Systems, Cambridge University Press, Cambridge 1995.

[13] P.W. Anderson, Phys. Rev. B 23, 4828 (1981). 